\title{
Orthopaedic Women of India: Impediments to Their Growth
}

\author{
Vrisha Madhuri $^{1}$ (]) Nabila Khan ${ }^{1}$
}

Received: 25 April 2020 / Accepted: 27 April 2020

(c) Indian Orthopaedics Association 2020

There is increasing interest in the role of women in Orthopaedics and their concerns. In some western countries, women in Orthopaedics constitute about 5\% and trainees form about $15 \%$ of the workforce [1]. In contrast, in India, they form about $1 \%$ of the Orthopaedic workforce [2]. With the focus on gender equality and making the working environment more conducive for Orthopaedic women, we have assessed the views, needs and challenges for trainees, junior and senior consultants and leaders.

All women Orthopaedic surgeons were invited to join by their peers to form an Indian Women Orthopaedics group. The database of those who had joined was created to get the baseline information of women in Orthopaedics and the data relating to age, geographical distribution, academic qualification and position held were obtained by soliciting these details. The issues faced by women Orthopaedic surgeons were identified after a literature survey, discussion with male and female peers and personal experiences. A questionnaire was created to assess the need for women in Orthopaedics, ease of getting into training and completing training programmes, promotions and managing Orthopaedic work. The set of questionnaires also assessed the challenges of work-family balance, family planning, and gender bias from patients, junior and senior colleagues, hospital staff and administrators. The challenging aspects such as pressure from work colleagues, training, physical ability to carry out work and losing the motivation were also explored. Those with negative responses were contacted by phone to obtain additional supporting information. The data were tabulated and expressed as percentages.

The database of the Women's Orthopaedic group showed that we had 221 women registered as group members at the time of conducting this survey. Of these, 162 were younger than 40 years. Trainees and fellows constituted one hundred

Vrisha Madhuri

madhuriwalter@cmcvellore.ac.in

1 Department of Pediatric Orthopedics, Christian Medical College, Vellore, India and twenty. The largest numbers were from Telangana and Andhra Pradesh. More than $90 \%$ held a Master's qualification. Only five held leadership positions.

Responses were obtained from 120 women. Need for more women in Orthopaedics was felt by $95 \%$; however, $25 \%$ had difficulty in getting into a training programme.

Discrimination in promotion was felt by two-third of the women and $95 \%$ noted gender bias. The work-life balance was challenging for $40 \%$, and the inability to achieve full working potential was felt by $60 \%$. Women were expected to maintain higher standards according to $75 \%$ of the responders. Family life and planning was affected for nearly half the women and two-third felt the lack of maternity support. Those wanting to leave the specialty constitute $11 \%$ even though all felt competent to do Orthopaedics.

Even in countries like USA, where the women trainees had achieved parity with their male colleagues in medical discipline by 2001 [3], the Orthopaedics trainees represent less than $14 \%$ of the Orthopaedic trainees in the year 2016-2017 [4]. This is partly attributed to lack of female Orthopaedic surgeons in higher ranks in the medical schools and, therefore, the lack of mentorship for them in the discipline [5, 6]. The work-life balance considered as important deterrent in $78 \%$ of the respondent in USA [5] was considered as a perceived difficulty only in $40 \%$ of our Orthopaedic surgeons and, thus, not seen as a major issue. It is worrying that in this survey, we elicited that among those few who have joined the discipline, many regret the decision even though they feel competent to carry out their work. Discrimination and inability to achieve full potential are a matter for concern. To address these, changes in behaviour and culture will require education about discriminatory behaviour, sexual harassment, non-intimidatory teaching methods and how to deliver feedback appropriately [7].

The survey suggests that the women Orthopaedic surgeons in India are in a very small minority and face genderbased issues. Given their capacity to contribute to Orthopaedics, it is necessary to evaluate and make changes to people's perceptions, attitudes and work environment. This 
paper is the first to highlight issues relating to Indian women in Orthopaedics.

Acknowledgements We acknowledge Dr. Rujuta Mehta who was instrumental in creating the WOICE group to give voice to Indian women and other WOICE members for their suggestions, providing information relating to the group and contributing to the survey.

\section{Compliance with Ethical Standards}

Conflict of interest None of the authors have any conflict of interest to declare or any financial disclosure.

Ethical standard statement This article does not contain any studies with human or animal subjects performed by the any of the authors.

Informed consent For this type of study informed consent is not required.

\section{References}

1. Demaio, M., (2019). Making the case (again) for gender equity. AAOS Now. https://www.aaos.org/aaosnow/2019/jun/youraaos/ youraaos $05 /$
2. Jain, A. K. (2016). Current state of orthopedic education in India. Indian J Orthopaedics, 50(4), 341-344. https://doi. org/10.4103/0019-5413.185586.

3. Blakemore, L. C., Hall, J. M., \& Biermann, J. S. (2003). Women in surgical residency training programs. JBJS, 85(12), 2477-2480.

4. Chambers, C. C., Ihnow, S. B., Monroe, E. J., \& Suleiman, L. I. (2018). Women in Orthopaedic surgery: Population trends in trainees and practicing surgeons. JBJS, $100(17)$, e116.

5. Rohde, R. S., Wolf, J. M., \& Adams, J. E. (2016). Where are the women in Orthopaedic surgery? Clinical Orthopaedics and Related Research, 474(9), 1950-1956.

6. Miller, E. K., \& LaPorte, D. M. (2015). Barriers to women entering the field of orthopedic surgery. Orthopedics, 38(9), 530-533.

7. Crebbin, W., Campbell, G., Hillis, D. A., \& Watters, D. A. (2015). Prevalence of bullying, discrimination and sexual harassment in surgery in Australasia. ANZ Journal of Surgery, 85(12), 905-909.

Publisher's Note Springer Nature remains neutral with regard to jurisdictional claims in published maps and institutional affiliations. 